\title{
Improving head-up display with waveguides and holographic optical elements
}

Colton M. Bigler, Micah S. Mann, Craig Draper, Arkady Bablumyan, Pierre-Alexandre Blanche

Colton M. Bigler, Micah S. Mann, Craig Draper, Arkady Bablumyan, PierreAlexandre Blanche, "Improving head-up display with waveguides and holographic optical elements," Proc. SPIE 10944, Practical Holography XXXIII: Displays, Materials, and Applications, 109440H (27 February 2019); doi: $10.1117 / 12.2507575$ 


\title{
Improving head-up display with waveguides and holographic optical elements
}

\author{
Colton M. Bigler ${ }^{\mathrm{a}}$, Micah S. Mann ${ }^{\mathrm{a}}$, Craig Draper ${ }^{\mathrm{a}}$, Arkady Bablumyan ${ }^{\mathrm{b}}$, and \\ Pierre-Alexandre Blanche ${ }^{a, b}$ \\ ${ }^{a} \mathrm{U}$ of A, College of Optical Sciences, 1630 E University Blvd, Tucson, AZ 85721, USA \\ ${ }^{\mathrm{b}}$ TIPD LLC, 502 Via Del Monte, Oceanside, CA 92058, USA
}

\begin{abstract}
Head-up displays offer ease-of-use and safety advantages over traditional head-down displays when implemented in aircraft and vehicles. Unfortunately, in the traditional head-up display projection method, the size of the image is limited by the size of the projection optics. In many vehicular systems, the size requirements for a large field of view head-up display exceed the space available to allocate for these projection optics. Thus, an alternative approach is needed to present a large field of view image to the user. By using holographic optical elements affixed to waveguides, it becomes possible to reduce the size of the projection system, while producing a comparatively large image. Additionally, modulating the diffraction efficiency of some of the holograms in the system presents an expanded viewing eyebox to the viewer. This presentation will discuss our work to demonstrate a magnified far-field image with an in-line two-dimensional eyebox expansion. It will explore recording geometries and configurations and will conclude by discussing challenges for future implementation.
\end{abstract}

Keywords: Holography, Head-Up Display, Holographic Optical Element, Diffraction, Waveguide, Field of View

\section{INTRODUCTION}

The term head-up display (HUD) refers to a display of instrument readings in an aircraft or vehicle that can be seen without lowering the eyes, typically through being projected on to the windscreen or visor. ${ }^{1}$ Functionally, this means that, for a vehicle using a HUD system, relevant safety, status, and control information can be displayed within the same field of view (FOV) the user would typically use to look at the external environment. This offers users a number of advantages over traditional dash-mounted or head-down display (HDD) designs, including shorter accommodation times and increased eyes-forward time, ${ }^{2,3}$ both of which translate to enhanced situational awareness and faster reaction time by users. ${ }^{4-6}$

Traditionally, HUD systems have been laid out as shown in Fig. 1, where light from a source is encoded with an image and propagated through a collection of relay optics toward a transparent, partially reflective combiner. The combiner reflects this light back to the user. ${ }^{7-12}$ The choice of relay optics in the system can locate the projected symbology in the observer's far field. ${ }^{13,14}$ This display system presents relevant control information to the vehicle's operator, while still allowing light from beyond the combiner to propagate to the observer.

Despite the advantages that HUD presents to drivers and pilots, it has not been widely implemented in either automotive or aviation applications. This is due, in part, to the small FOV available with the traditional system. In order to project a larger image, the size of the relay optics needs to increase. Unfortunately, this size increase to the relay optics, and thus the packaged volume, quickly becomes unfeasible for most vehicular applications. ${ }^{15-17}$ Additionally, the traditional projection system suffers from a small eyebox, which describes the area over which an observer can see the unvignetted image.

To address these issues, recent research has explored the use of diffractive optics and holographic optical elements (HOEs) to achieve image magnification and pupil expansion. ${ }^{18-24}$ In these systems, a HOE couples light into a waveguide, where it propagates due to total internal reflection (TIR) until it interacts with other

Further author information: (Send correspondence to C.M.B.)

C.M.B.: E-mail: cbigler@optics.arizona.edu, Telephone: 15206263416

Practical Holography XXXIII: Displays, Materials, and Applications, edited by Hans I. Bjelkhagen,

V. Michael Bove, Proc. of SPIE Vol. 10944, 109440H · (C) 2019 SPIE

CCC code: $0277-786 \mathrm{X} / 19 / \$ 18 \cdot$ doi: $10.1117 / 12.2507575$

Proc. of SPIE Vol. 10944 109440H-1 


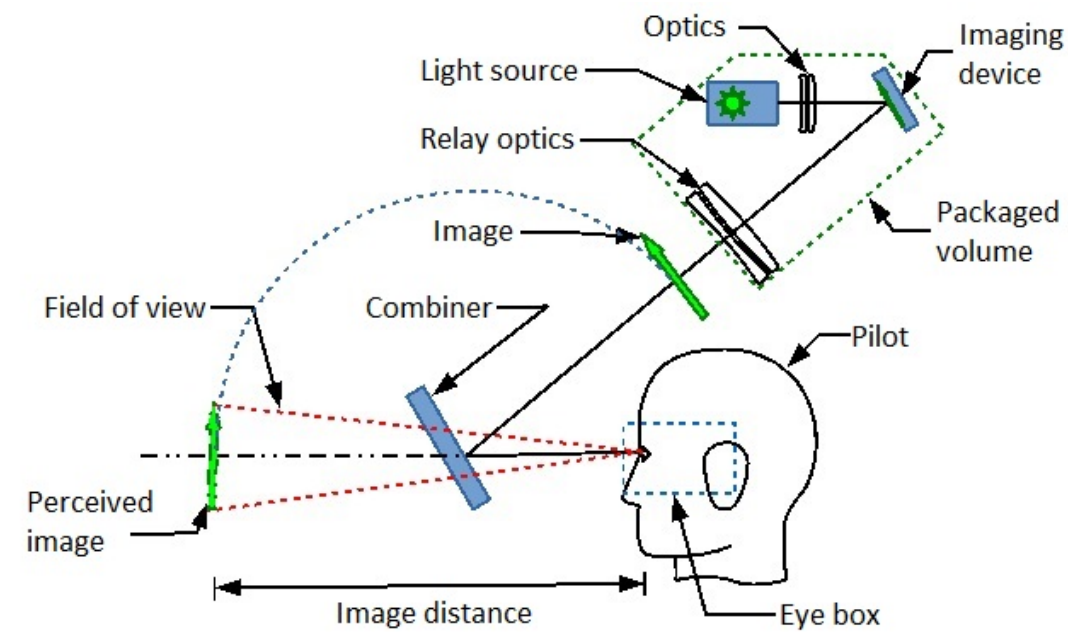

Figure 1: Conventional HUD system schematic. Light from the source is encoded with a relevant image by the imaging device. This image passes through the relay optics so that it is located in the far field. This far field image is partially reflected off of the combiner and presented to the observer in an area called the eye box.

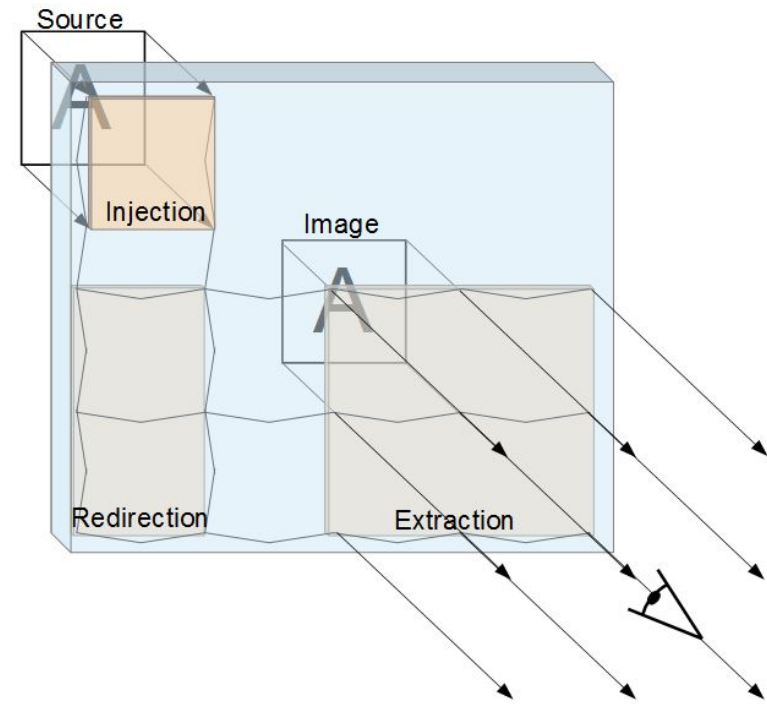

Figure 2: Light from an outside source is projected through the waveguide HOE system to present an image to the observer over an expanded eyebox. Successive HOEs increase the exit pupil size, vertically then horizontally.

HOEs, each of which might modify the beam or redirect it out of the waveguide toward an observer. Fig. 2 provides an example of how these systems might work: An 'injection' HOE redirects the light from a source down the length of a waveguide to a 'redirection' HOE, which magnifies the exit pupil vertically and sends the light laterally along the length of the waveguide. The diffraction efficiency (DE) of the 'redirection' HOE is modulated to achieve uniform intensity across the entire beam profile as it propagates away to the 'extraction'. The 'extraction' HOE achieves horizontal pupil expansion with the same modulated DE technique and redirects the light out of the waveguide to the observer.

Previous research efforts have demonstrated an "L" shaped 2D pupil expansion. ${ }^{24}$ However, in this geometry, the 'redirection' HOE in Fig. 2 serves as a limiting factor on the FOV, as the angular bandwidth of the holograms is taxed by the $90^{\circ}$ beam redirection. In this paper, we propose an in-line vertical pupil expansion to increase the effective FOV presented through the system. We use an Optic Studio model to demonstrate the feasibility of such a system, achieving a FOV of $11^{\circ} \times 11^{\circ}$, though this seems to be limited by the size of the surface relief grating (SRG) and the number of segments available to the program for the ray trace. Subsequent research 
efforts will aim to refine the computer model and create a physical demonstrator of the principle.

\section{IN-LINE 2D PUPIL EXPANSION}

In this paper, we share a novel approach to achieve pupil expansion in a waveguide HUD system with inline HOEs. In this system, light incident on an 'injection' HOE propagates down the length of a waveguide, where it interacts with a SRG, which splits the light into \pm 1 and 0-diffraction orders. Subsequent interactions of the diffracted light with the SRG again splits the light into a '0-order', which follows the initial propagation direction before interacting with the grating and ' \pm 1 ' orders, which continue in the initial propagation direction. The '0-order' diffraction extends the area of interaction for the light propagating along the length of the waveguide. This interaction vertically expands the eyebox of the system. Horizontal pupil expansion is achieved by encoding a variable DE across the length of the extraction so that the amount of light extracted from any point along the length of the waveguide is the same intensity. ${ }^{23}$ Figs. 3 and 4 demonstrate front and isometric views of the system, respectively. Note that some light propagates in the direction of the ' \pm 1 -orders', which is not directed to the observer and is, therefore, wasted in the existing system.
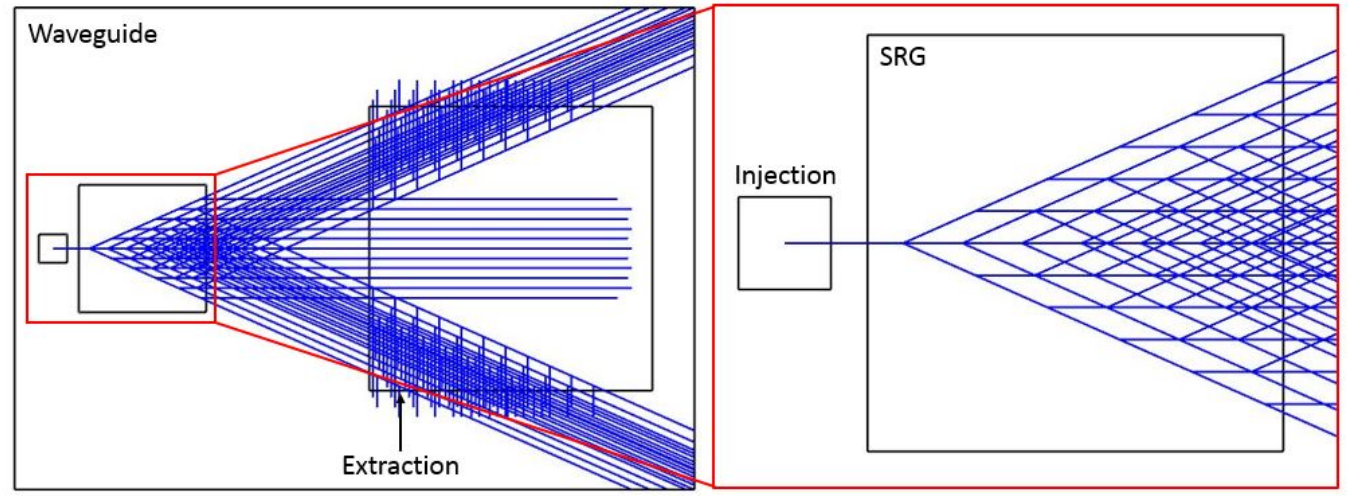

Figure 3: Front view of the in-line pupil expansion system. Light from the source is redirected into the waveguide by the injection. Interacting with the SRG causes the light to split into three diffraction orders. Subsequent interactions with the SRG cause some light to be diffracted back to the initial propagation direction, while the remainder of the light continues on in the direction determined by the initial interaction's ' \pm 1 diffraction orders'.

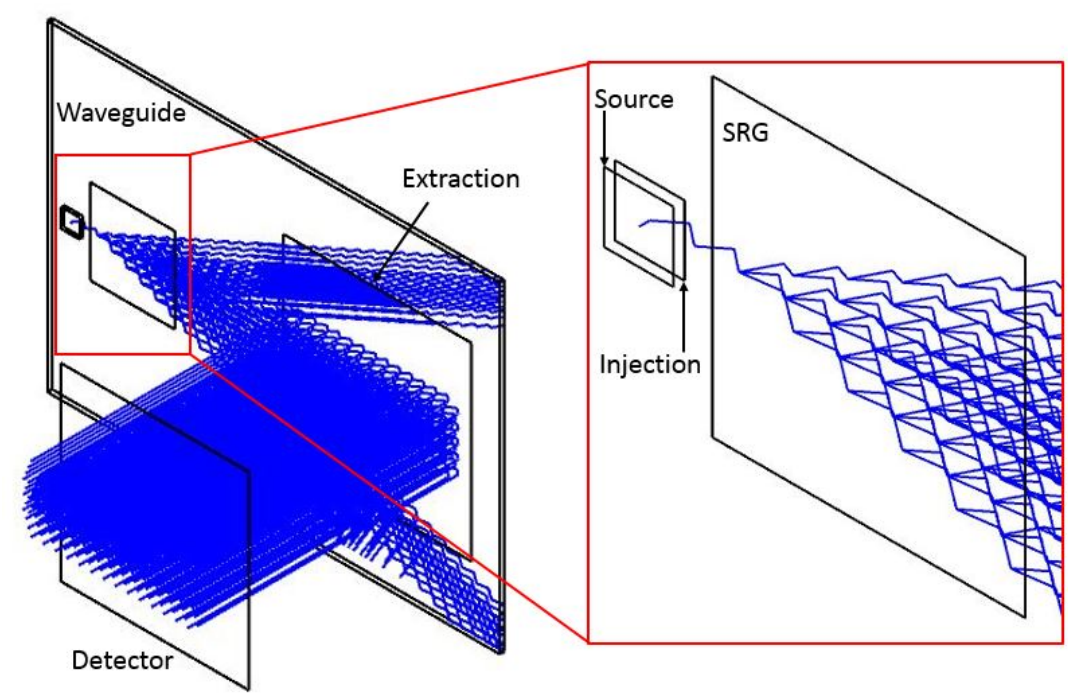

Figure 4: Isometric view of the in-line pupil expansion system. Interaction with a variable DE extraction presents the expanded ' 0 -order' to the observer, while the ' \pm 1 orders' are diffracted outside of the ideal viewing angle. 


\subsection{Injection}

The injection HOE is designed to redirect incident light beyond the critical angle so that the light continues propagating within the waveguide due to Total Internal Reflection (TIR). For glass with an index of 1.5, this critical angle is $41.8^{\circ}$. We chose the redirection angle of the injection hologram such that normally incident light propagates between the critical angle and a maximum angle determined by the size of the injection hologram and the thickness of the waveguide. The maximum propagation angle is given by $\tan \left(\theta_{\max }\right)=\frac{w / 2}{t}$ where $t$ is the waveguide thickness and $w$ is the width of the injection hologram. For our system with an injection hologram width of 0.5 " and a thickness of 0.125 ", the maximum propagation angle is $63.4^{\circ}$. The injection is recorded at the angle between these two extremes, for an injection hologram that redirects normally incident light at $52.6^{\circ}$.

\subsection{Extraction}

The extraction HOE is designed to diffract incident light propagating within the waveguide at the angle calculated for the injection hologram diffraction $\left(52.6^{\circ}\right)$ so that it propagates perpendicular to the waveguide surface. It is recorded with variable $\mathrm{DE}$ along the length to achieve uniform intensity across the entire extraction. In the case of this model, there are eight HOE segments with DE increasing from $1 / 8$ to 1 along the length of the HOE according to $\eta_{n}=\frac{1}{N-n+1}$ where $n$ is the current segment number, $\eta_{n}$ is the DE of the $n^{\text {th }}$ element, and $N$ refers to the total number of segments. The real HOE might have a gradient DE curve across its length, as opposed to the step function available in the computer simulation.

\subsection{Surface relief grating}

The injection and extraction HOE pair works to achieve horizontal pupil expansion, as shown in Fig.5a, where a thin portion of the projected image is visible. Moving the observation point vertically will shift the projected strip along the injected image, but will not increase the FOV.

To increase the FOV vertically, we added another diffractive feature to our system: an SRG that diffracts light into three diffraction orders while the light propagates laterally within the waveguide. We chose to use an SRG instead of a HOE because of the thickness of the feature and the fact that it diffracts light into multiple orders. This element was created in Zemax with the Lenslet Array tool, using a grating spacing of 1 line per micron. Figs. 5b and 5c show that the FOV is vertically expanded with an attached SRG. The SRG in Fig. 5b is half the size of that shown in Fig. 5c, meaning that there are not enough interactions between the propagating light and the SRG to expand the FOV so that the system shows the entire image to the observer.

\section{NEXT STEPS}

Having successfully demonstrated a simulation that predicts the viability of the in-line 2D pupil expansion effect, our next research efforts will seek to develop a physical demonstrator in the geometry discussed in Sec. 2. We have recorded a successful injection/extraction HOE pair, as shown in Fig. 6, and are in the process of recording a corresponding SRG using lithographic printing methods. One area that bears continued investigation is that of a variable depth along the vertical segments of the SRG, which might reduce the amount of wasted light sent into the ' \pm 1 -order' directions.

\section{CONCLUSION}

HUD has the potential to improve driver and pilot safety if successfully implemented in commercial vehicles. However, the FOV of current HUD designs has prevented this technology from being implemented as anything other than a novelty. In this paper, we explore existing HUD technologies and the ways they attempt to improve the FOV of the system. We begin by introducing a traditional projection geometry with a combiner and transitioned to waveguide HOE-assisted HUDs. The main body of this paper explored a Zemax OpticStudio model of a new waveguide HOE geometry that achieves FOV and eyebox expansion with a SRG overlaid on the 1D pupil expansion configuration. Our design has a FOV of $11^{\circ} \times 11^{\circ}$. We look forward to completing this research effort in the near future with a physical demonstrator of the principles demonstrated in the model. 


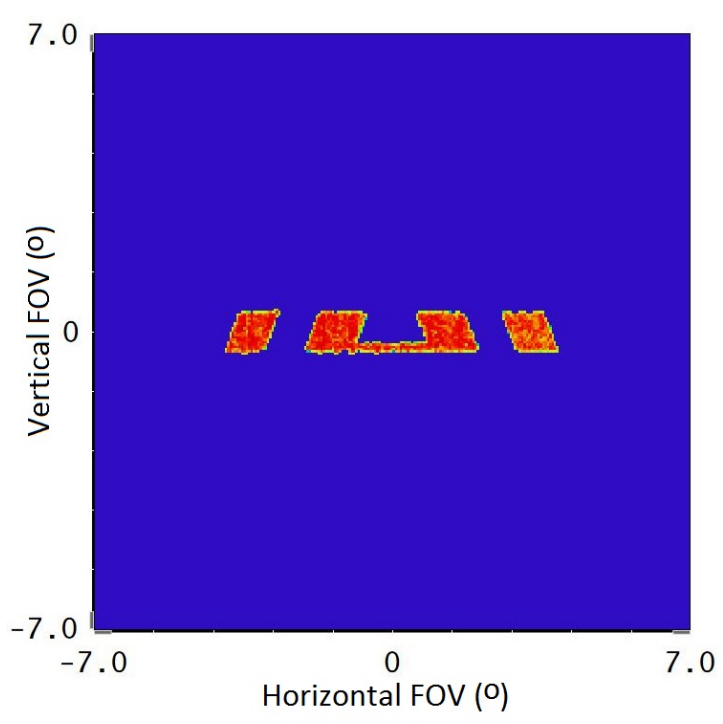

(a) Without SRG, FOV is $8^{\circ} \times 0.8^{\circ}$

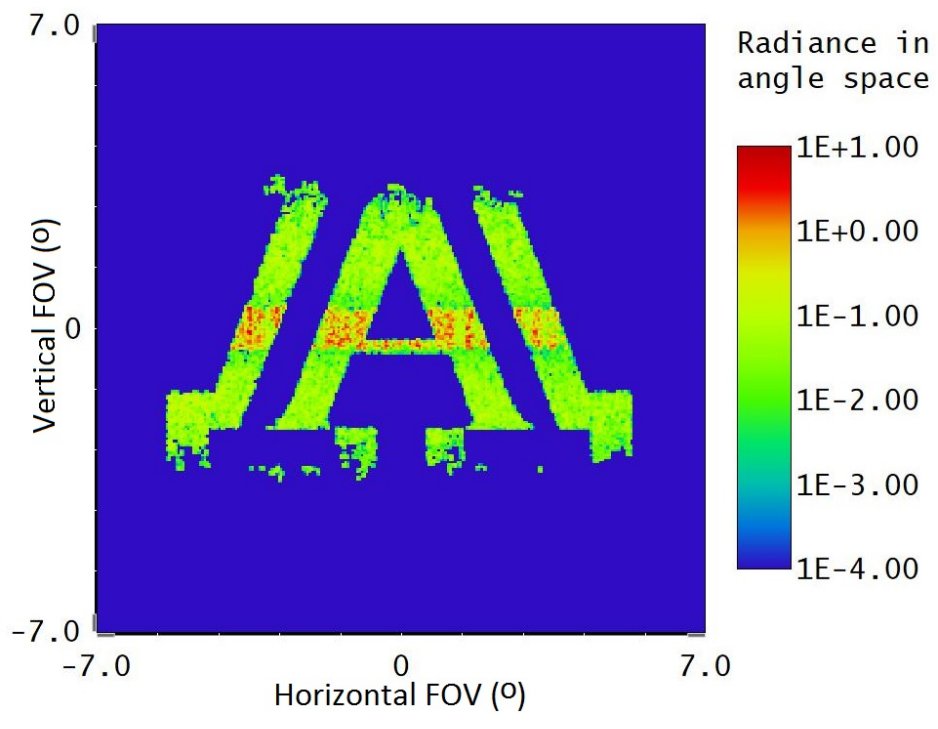

(b) With limited size SRG, FOV is $11^{\circ} \times 6^{\circ}$

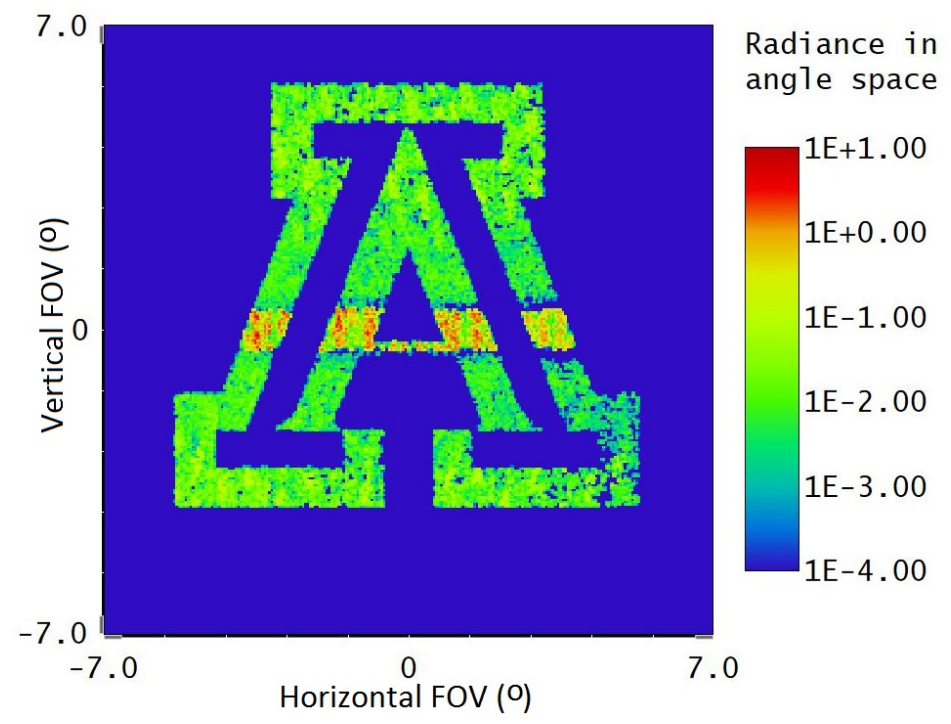

(c) With full-size SRG, FOV is $11^{\circ} \times 11^{\circ}$

Figure 5: FOV of system show in Figs. 3 and 4 without a SRG, with a small SRG, and with a larger SRG. The viewing distance is $150 \mathrm{~mm}$ on a $4 \mathrm{~mm}$ detector. Horizontal pupil expansion takes place in all cases, but the SRG is necessary for vertical pupil expansion. The size of the SRG determines the maximum FOV.

\section{ACKNOWLEDGMENTS}

The authors would like to thank Ben Cromey for his help with designing and troubleshooting the OpticStudio model presented here.

\section{REFERENCES}

[1] "Definition of head-up display." Oxford University Press, https://en.oxforddictionaries.com/ definition/head-up_display (2018). (Accessed: 12 December 2018).

[2] Liu, Y.-C. and Wen, M.-H., "Comparison of head-up display (hud) vs. head-down display (hdd): Driving performance of commercial vehicle operators in taiwan," Int. J. Hum.-Comput. Stud. 61, 679-697 (Nov. 2004). 


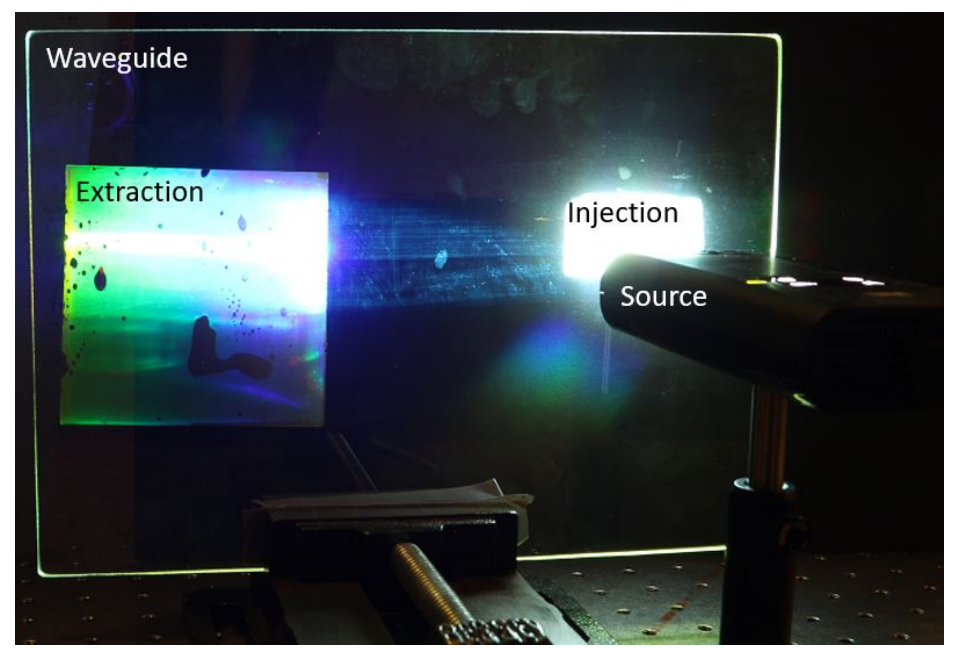

Figure 6: Recorded demonstrator of injection and extraction HOEs.

[3] Ablassmeier, M., Poitschke, T., Wallhoff, F., Bengler, K., and Rigoll, G., "Eye gaze studies comparing head-up and head-down displays in vehicles," in [2007 IEEE International Conference on Multimedia and Expo], 2250-2252 (July 2007).

[4] Wickens, C. and Ververs, P., "Allocation of attention with head-up displays," tech. rep., Aviation Research Laboratory, Institute of Aviation (November 1998).

[5] Fadden, S., Wickens, C. D., and Ververs, P., "Costs and benefits of head up displays: An attention perspective and a meta analysis," in [SAE Technical Paper], SAE International (10 2000).

[6] Horrey, W. J., Wickens, C. D., and Alexander, A. L., "The effects of head-up display clutter and in-vehicle display separation on concurrent driving performance," Proceedings of the Human Factors and Ergonomics Society Annual Meeting 47(16), 1880-1884 (2003).

[7] Upatnieks, J., "Compact head-up display," (Dec. 8 1987). US Patent 4,711,512.

[8] Wood, R. B. and Hayford, M. J., "Holographic and classical head up display technology for commercial and fighter aircraft," Proc. SPIE 0883, 36-52 (1988).

[9] Kato, H., Ito, H., Shima, J., Imaizumi, M., and Shibata, H., "Development of hologram head-up display," in [SAE Technical Paper], SAE International (02 1992).

[10] Kalmanash, M. H., "Digital huds for tactical aircraft," Proc. SPIE 6225, 62250L-62250L-8 (2006).

[11] Peng, H., Cheng, D., Han, J., Xu, C., Song, W., Ha, L., Yang, J., Hu, Q., and Wang, Y., "Design and fabrication of a holographic head-up display with asymmetric field of view," Appl. Opt. 53, H177-H185 (Oct 2014).

[12] Han, J., Liu, J., Yao, X., and Wang, Y., "Portable waveguide display system with a large field of view by integrating freeform elements and volume holograms," Opt. Express 23, 3534-3549 (Feb 2015).

[13] Coni, P., Hourlier, S., Gueguen, A., Servantie, X., and Laluque, L., "50-3: A full windshield head-up display using simulated collimation," SID Symposium Digest of Technical Papers 47(1), 684-687 (2016).

[14] Coni, P., Hourlier, S., Servantie, X., Laluque, L., and Gueguen, A., "A 3d head up display with simulated collimation," in [SAE Technical Paper], SAE International (09 2016).

[15] Vallance, C. . H., "The approach to optical system designs for aircraft head up displays," Proc. SPIE 0399, 15-25 (1983).

[16] Bartlett, C. T., Busbridge, M. L., and Horton, O. T., "Considerations of a head-up display field of view," Proc. SPIE 4712, 468-479 (2002).

[17] Wisely, P. L., "Head up and head mounted display performance improvements through advanced techniques in the manipulation of light," Proc. SPIE 7327, 732706-732706-10 (2009).

[18] Cox, J. A., Fritz, T. A., and Werner, T. R., "Application and demonstration of diffractive optics for headmounted displays," Proc. SPIE 2218, 32-40 (1994). 
[19] Cameron, A. A., "Optical waveguide technology and its application in head-mounted displays," Proc. SPIE 8383, 83830E-83830E-11 (2012).

[20] Harbour, S. D., "Three-dimensional system integration for hud placement on a new tactical airlift platform: design eye point vs. hud eye box with accommodation and perceptual implications," Proc. SPIE 8383, $83830 \mathrm{~V}-83830 \mathrm{~V}-7$ (2012).

[21] Homan, M., "The use of optical waveguides in head up display (hud) applications," Proc. SPIE 8736, 87360E-87360E-14 (2013).

[22] Wilmington, I. K. and Valera, M. S., "Paper no 18.2: Waveguide-based display technology," SID Symposium Digest of Technical Papers 44, 278-280 (2013).

[23] Bigler, C. M., Blanche, P.-A., and Sarma, K., "Holographic waveguide heads-up display for longitudinal image magnification and pupil expansion," Appl. Opt. 57, 2007-2013 (Mar 2018).

[24] Draper, C., Bigler, C. M., Mann, M., Sarma, K., and Blanche, P.-A., "Holographic waveguide head-up display with 2-d pupil expansion and longitudinal image magnification," Appl. Opt. . Accepted for publication. 